\title{
Is robotic utilization associated with increased minimally invasive colorectal surgery rates? Surgeon-level evidence
}

\author{
Carla F. Justiniano $^{1}$ D $\cdot$ Adan Z. Becerra $^{2} \cdot$ Anthony Loria $^{1} \cdot$ Zhaomin Xu $^{1} \cdot$ Christopher T. Aquina $^{3} \cdot$ Larissa K. Temple $^{1}$. \\ Fergal J. Fleming ${ }^{1}$
}

Received: 15 February 2021 / Accepted: 3 January 2022 / Published online: 13 January 2022

(c) The Author(s), under exclusive licence to Springer Science+Business Media, LLC, part of Springer Nature 2022

\begin{abstract}
Background It is unclear whether robotic utilization has increased overall minimally invasive colorectal surgery rates or if robotics is being adopted instead of laparoscopy. The goal was to evaluate whether increasing robotic surgery utilization is associated with increased rates of overall colorectal minimally invasive surgery.

Methods The Statewide Planning and Research Cooperative System (New York) was used to identify patients undergoing elective colectomy or proctectomy from 2009 to 2015. Individual surgeons were categorized as having increasing or nonincreasing robotic utilization (IRU or non-IRU, respectively) based on the annual increase in the proportion of robotic surgery performed. The odds of surgical approach across the study period were evaluated with multinomial regression.

Results Among 72,813 resections from 2009 to 2015, minimally invasive-surgery increased $(47-61 \%, p<0.0001)$. For colectomy, overall minimally invasive-surgery rates increased $(54-66 \%, p<0.0001)$, laparoscopic remained stable $(53-54 \%)$, and robotics increased (1-12\%). For proctectomy, overall minimally invasive-surgery rates increased $(22-43 \%, p<0.0001)$, laparoscopic remained stable (20-21\%), and robotics increased (2-22\%). Over the study period, 2487 surgeons performed colectomies. Among 156 surgeons with IRU for colectomies, robotics increased (2-29\%), while laparoscopy decreased (67-44\%), and open surgery decreased (31-27\%). Overall, surgeons with IRU performed minimally invasive colectomies $73 \%$ of the time in 2015 versus $69 \%$ in 2009. Over the study period, 1131 surgeons performed proctectomies. Among 94 surgeons with IRU for proctectomies, robotics increased (3-42\%), while laparoscopy decreased (25-15\%), and open surgery decreased (73-44\%). Overall, surgeons with IRU performed minimally invasive proctectomy 56\% of the time in 2015 versus $27 \%$ in 2009. Patients in the latter study period had $57 \%$ greater odds of undergoing robotic surgery.

Conclusions Overall, minimally invasive colorectal resections increased from 2009 to 2015 largely due to increasing robotic utilization, particularly for proctectomies.
\end{abstract}

Keywords Robotic surgery $\cdot$ Colorectal surgery $\cdot$ Colectomy $\cdot$ Proctectomy

The corresponding abstract was accepted for a quick shot podium presentation at the ASCRS 2020 Annual Scientific Meeting in Boston, MA which was virtually presented due to the COVID-19 pandemic.

Carla F. Justiniano

carla.justiniano.md@gmail.com

1 Surgical Health Outcomes \& Research Enterprise (SHORE), Department of Surgery, University of Rochester Medical Center, 601 Elmwood Avenue, Box SURG, Rochester, NY 14642, USA

2 Department of Surgery, Rush University Medical Center, Chicago, IL, USA

3 Division of Surgical Oncology, Department of Surgery, The Ohio State Medical Center, Columbus, OH, USA
Colon and rectal resections are among the most common operations performed in the United States [1]. Since the advent of minimally invasive techniques in the early 1990's, there has been a concerted effort to critically assess the role of minimally invasive-surgery (MIS) in colorectal resections [2-4]. By the mid 1990's, 25 randomized controlled trials were published addressing whether MIS was superior to open techniques. A Cochrane Library meta-analysis demonstrated that compared to open colorectal resections, MIS was associated with reduced post-operative pain, faster resolution of post-operative ileus, shortened hospitalizations, and reduced morbidity [5]. Over the past 2 decades, many studies have built on this early evidence supporting MIS for 
the treatment of benign and malignant colorectal pathology [6-10].

Studies evaluating minimally invasive colorectal surgery have largely focused on colectomies alone or rarely distinguish them from proctectomies [11, 12]. Studies of proctectomies suggest that there has been slower adoption of MIS compared to colectomies [13]. Consequently, some experts have suggested that a potential route toward increasing value and outcomes in rectal surgery could be through expanded robotic use as the robot has technical advantages in the constraints of the pelvis [14]. At the surgeon-level, the trends of robotic utilization for colon and rectal surgery have not been studied beyond small samples. Whether robotic surgery increases MIS for colon, and especially rectal, resections remains unclear.

A population-based, all-payer study was performed to evaluate whether an overall increase in MIS has occurred for colectomies and proctectomies over time. We aimed to determine whether robotic surgery is being increasingly adopted over open techniques or whether an intra-MIS migration has occurred with cases shifting from laparoscopic to robotic. A priori, our hypothesis was that for colectomies, robotic surgery has largely replaced laparoscopy resulting in a modest increase in the overall rate of MIS. Conversely for proctectomies, we hypothesized that robotic operative approaches have supplanted both open and laparoscopic approaches resulting in significantly increased rates of minimally invasive proctectomies.

\section{Materials and methods}

\section{Data source and study population}

Adults who underwent elective colectomy or proctectomy between 2009 and 2015 in the Statewide Planning and Research Cooperative System (SPARCS) database were included. This database is an all-payer, population-based, hospital reporting system from the New York Department of Health which captures patient-level data on all non-Veteran Affairs hospitals. Unique hospital and surgeon identifiers are captured for each admission; numerous previous studies have described the unique identifiers [10, 15-17]. SPARCS was linked to the American Medical Association Physician Masterfile and American Board of Medical Specialties database (Medical Marketing Service, Inc, Woodale, IL) to determine surgeon board certification and duration of practice. Linkage to the American Hospital Association database from the Council of Teaching Hospitals was performed to attain urban or rural location and major academic status as defined by the Council of Teaching Hospitals.

\section{Patient-, surgeon- and hospital-level factors}

Age, sex, race, receipt of Medicaid insurance and comorbidity burden were captured for each patient. Comorbidities were captured as previously described and the Van Walraven Elixhauser modification was used to compare comorbidity as derived from administrative data ICD codes; the disease burden was classified as low-, moderate- and high-risk [18-21]. In addition to surgeon-years in practice and board certification, surgeon volume for colectomy (low $<12$, medium $12-32$, high $\geq 33$ ) and proctectomy (low $<5$, medium $6-18$, high $\geq 19$ ) was calculated and categorized by tertile. In addition to hospital rural or academic status, hospital volume for colectomy (low $<97$, medium $98-218$, high $\geq 219$ ) and proctectomy (low $<34$, medium $34-87$, high $\geq 88$ ) was calculated and categorized by tertile.

\section{Outcome measures}

Surgeons were classified as having increasing or nonincreasing robotic utilization (IRU or non-IRU, respectively) for colectomy and proctectomy. Surgeons with IRU had an overall increasing trend in robotic utilization over time whereas non-IRU surgeons did not have an increasing trend in robotic utilization over time. The primary outcome was change in operative approach rates over time; this was further explored among surgeons with IRU. Factors associated with operative approach were also examined.

\section{Statistical analysis}

Linear regression models were used to estimate the association between year of surgery and yearly rates of robotic surgery for each surgeon. The slope for year of surgery represents the average annual change in proportional use of robotic surgery. If the slope averaged over all years was positive, surgeons were classified as having IRU, whereas surgeons who did not have a positive average slope were classified as having non-IRU [22]. For example, if a surgeon had 1 year of increased proportional use of robotic surgery, 1 year of decreased proportional use of robotics but a smaller percentile change than the increase, and then otherwise stable usage, then the overall slope would still remain positive and this surgeon would be considered to have IRU. Surgeon characteristics across IRU or non-IRU groups were evaluated using the $\chi^{2}$ test, two-sided $t$ test and Kruskal-Wallis test, as appropriate to the data. Similarly, patient characteristics were compared by operative approach. Bayesian multinomial regression, accounting for clustering at the hospitaland surgeon-level was used to estimate the odds of surgical approach at the patient-level. The R package MCMCglmm was used to perform the multinomial regression analysis $(\mathrm{R}$ 
version 3.5.1; R Foundation, Vienna, Austria). All other data manipulation and analyses were performed on SAS software version 9.4 (SAS Institute, Cary, NC, USA). The study was approved by the University of Rochester (IRB\#00054886) Institutional Review Board.

\section{Results}

Between 2009 and 2015, 72,813 patients met eligibility criteria (Fig. 1 and Table 1). Overall, 2487 surgeons (156 or $6 \%$ had IRU for colectomy) performed 57,237 elective colectomies. Over the same period, 1311 surgeons (94 or $7 \%$ had IRU for proctectomy) performed 15,576 elective proctectomies. Thirty-seven percent of the colectomy IRU surgeons were board certified in colorectal surgery, and while this group tended to have had fewer years in practice, $29 \%$ still had greater than 21 years of experience (Table 2). Fifty-three percent of the proctectomy IRU surgeons were board certified in colorectal surgery, and while they tended to be less experienced, $28 \%$ still had over 21 years of experience. The majority of proctectomy IRU surgeons also were colectomy IRU surgeons; however, only $46 \%$ of colectomy IRU surgeons also had IRU for proctectomies. Among surgeons with IRU, the rate of conversion-to-open among robotic cases decreased from $5.5 \%$ in 2009 to $2.8 \%$ in 2015 ( $p$-value 0.1894).

For the entire cohort, the rate of minimally invasive colorectal resections increased from $47 \%$ in 2009 to $61 \%$ in 2015, $p<0.0001$. For colectomies, the rate of MIS increased from 54\% in 2009 to $66 \%$ in 2015 ( $p<0.0001)$. For colectomies, this overall increase in MIS was accompanied by increased rates of robotics from $1 \%$ in 2009 to $12 \%$ in 2015 and stable rates of laparoscopic colectomy at 53\% in 2009 and 54\% in 2015 ( $p<0.0001$, Fig. 2).

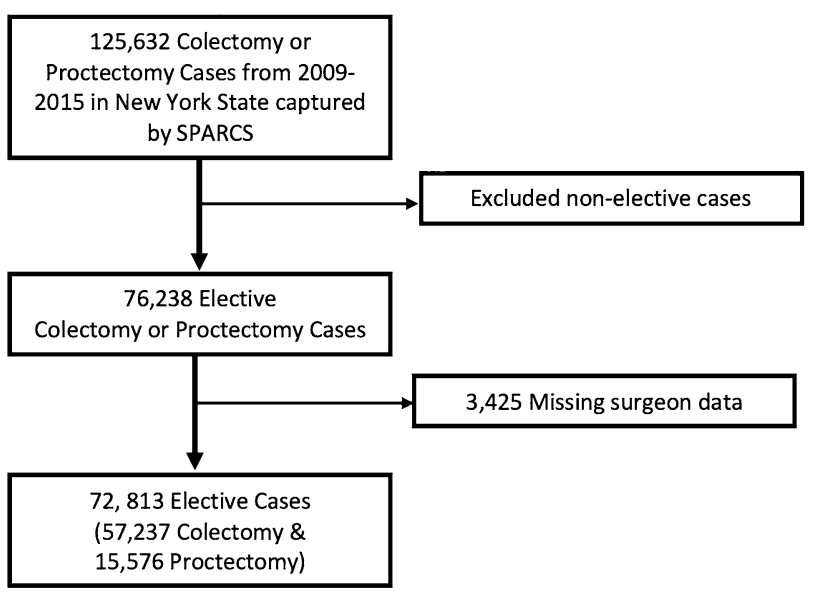

Similarly, for proctectomies, the overall MIS rate increased from 22 to $43 \%(p<0.0001)$. When classifying by type of MIS proctectomy, robotic use increased from 2\% in 2009 to $22 \%$ in 2015 while laparoscopy remain unchanged at $20 \%$ in 2009 and $21 \%$ in 2015 ( $p<0.0001$, Fig. 3).

Both surgeons with IRU and non-IRU for colectomy had increased rates of MIS over time, from 69 to $73 \%$ for IRU surgeons $(p=0.0006)$ and $48-61 \%$ for non-IRU surgeons $(p<0.0001)$. Among surgeons with IRU for colectomy $(n=156)$, the rate of robotic use increased from $2 \%$ in 2009 to $29 \%$ in 2015; meanwhile, there was a large decrease in the proportion of laparoscopic cases over time from 67 to $44 \%$ and a smaller decrease in the proportion of open cases $31-27 \%$ ( $p<0.0001$, Fig. 4$)$.

Both surgeons with IRU and non-IRU for proctectomy had increased rates of MIS overtime from 27 to $56 \%$ for IRU surgeons $(p<0.0001)$ and $20-31 \%$ for non- IRU surgeons $(p<0.0001)$. Among surgeons with IRU for proctectomies $(n=94)$, the rate of robotic use increased from $3 \%$ in 2009 to $42 \%$ in 2015; meanwhile, there was a small decrease in the proportion of laparoscopic cases over time from 25 to $15 \%$ and a large decrease in the proportion of open cases from 73 to $44 \%$ ( $p<0.0001$, Fig. 5).

In multivariable analysis, after controlling for patient-, surgeon- and hospital-level characteristics (Table 3), patients were more likely to undergo robotic surgery in recent years (2013-2015) as compared to earlier years (2009-2012). Compared to a robotic approach, the odds of laparoscopic surgery decreased in recent years by $76 \%$, and the odds of open surgery also decreased in recent years by $82 \%$. As compared to robotic surgery, patients age 75 or greater had $42 \%$ greater odds of receiving laparoscopic surgery and $65 \%$ greater odds of receiving open surgery. As compared to patients without Medicaid insurance, those who had Medicaid insurance had 52\% greater odds of receiving open surgery instead of robotic. The odds of operative approach did not significantly vary by race in multinomial analysis. As compared to robotic surgery, patients with medium and high comorbidity scores were $43 \%$ and $121 \%$, respectively, more likely to undergo open surgery. Patient who had operations by high volume surgeons were $71 \%$ less likely to undergo open surgery as compared to robotic, than patients who had surgery by low volume surgeons. Patients who had operations at higher volume hospitals, as compared to robotic surgery, were less likely to undergo laparoscopic and open surgery. As surgeon experience increases, the odds of performing laparoscopic surgery, as compared to robotic, significantly decreases (Fig. 6).

Fig. 1 Study population 
Table 1 Factors associated with operative approach

\begin{tabular}{|c|c|c|c|}
\hline & Robotic & Lap & Open \\
\hline \multicolumn{4}{|l|}{ Surgery year*^ } \\
\hline 2009-2012 & $1163(26.3 \%)$ & $21,398(59.1 \%)$ & $20,360(63.3 \%)$ \\
\hline 2013-2015 & $3263(73.7 \%)$ & $14,802(40.9 \%)$ & $11,827(36.7 \%)$ \\
\hline \multicolumn{4}{|l|}{ Patient age $* \wedge$} \\
\hline$\leq 60$ & $2292(51.8 \%)$ & $17,519(48.4 \%)$ & $14,285(44.4 \%)$ \\
\hline $61-74$ & $1450(32.8 \%)$ & $12,015(33.2 \%)$ & $10,584(32.9 \%)$ \\
\hline$\geq 75$ & $684(15.4 \%)$ & $6666(18.4 \%)$ & $7318(22.7 \%)$ \\
\hline Male patient ${ }^{* \wedge}$ & $2214(50.0 \%)$ & $17,099(47.2 \%)$ & $14,247(44.3 \%)$ \\
\hline \multicolumn{4}{|c|}{ Medicaid insurance* } \\
\hline No & $4290(96.9 \%)$ & $35,136(97.1 \%)$ & $30,718(95.4 \%)$ \\
\hline Yes & $136(3.1 \%)$ & $1064(2.9 \%)$ & $1469(4.6 \%)$ \\
\hline \multicolumn{4}{|l|}{ Patient race $* \wedge$} \\
\hline White & $3606(81.5 \%)$ & $28,190(77.9 \%)$ & $24,343(75.6 \%)$ \\
\hline Black & $298(6.7 \%)$ & $3043(8.4 \%)$ & $3279(10.2 \%)$ \\
\hline Other & $522(11.8 \%)$ & $4967(13.7 \%)$ & $4565(14.2 \%)$ \\
\hline \multicolumn{4}{|l|}{ Surgery*^ } \\
\hline Proctectomy & $1744(39.4 \%)$ & $3333(9.2 \%)$ & $10,499(32.6 \%)$ \\
\hline Colectomy & $2682(60.6 \%)$ & $32,867(90.8 \%)$ & $21,688(67.4 \%)$ \\
\hline \multicolumn{4}{|l|}{ ECS risk*^ } \\
\hline Low & $1442(32.6 \%)$ & $13,964(38.6 \%)$ & $8700(27.0 \%)$ \\
\hline Medium & $1888(42.7 \%)$ & $13,990(38.6 \%)$ & $13,000(40.4 \%)$ \\
\hline High & $1096(24.8 \%)$ & $8246(22.8 \%)$ & $10,487(32.6 \%)$ \\
\hline \multicolumn{4}{|c|}{ Surgeon Volume (tertile)*^ } \\
\hline Low & $299(6.8 \%)$ & $5553(15.3 \%)$ & $11,206(32.8 \%)$ \\
\hline Med & $935(21.1 \%)$ & $11,556(31.9 \%)$ & $11,414(35.5 \%)$ \\
\hline High & $3192(72.1 \%)$ & $19,091(52.7 \%)$ & $9567(29.7 \%)$ \\
\hline \multicolumn{4}{|c|}{ Hospital volume (tertile)*^ } \\
\hline Low & $648(14.6 \%)$ & $8418(23.3 \%)$ & $11,445(35.6 \%)$ \\
\hline Med & $1862(42.1 \%)$ & $12,458(34.4 \%)$ & $9805(30.4 \%)$ \\
\hline High & $1916(43.3 \%)$ & $15,324(42.3 \%)$ & $10,937(34.0 \%)$ \\
\hline \multicolumn{4}{|l|}{ Rural hospital*^ } \\
\hline No & $4359(98.5 \%)$ & $34,671(95.8 \%)$ & $30,225(93.9 \%)$ \\
\hline Yes & $67(1.5 \%)$ & $1529(4.2)$ & $1962(6.1 \%)$ \\
\hline \multicolumn{4}{|c|}{ Academic hospital ${ }^{* \wedge}$} \\
\hline No & $1820(41.1 \%)$ & $18,287(50.5 \%)$ & $15,972(49.6 \%)$ \\
\hline Yes & $2606(58.9 \%)$ & $17,913(49.5 \%)$ & $16,215(40.5 \%)$ \\
\hline \multicolumn{4}{|c|}{ Colorectal surgery boarded ${ }^{* \wedge}$} \\
\hline No & $1090(24.6 \%)$ & $19,367(53.5 \%)$ & $20,047(62.3 \%)$ \\
\hline Yes & $3336(75.4 \%)$ & $16,833(46.5 \%)$ & $12,140(37.7 \%)$ \\
\hline \multicolumn{4}{|c|}{ Surgeon-years of experience ${ }^{* \wedge}$} \\
\hline$<5$ years & $375(8.5 \%)$ & $4475(12.4 \%)$ & $2484(7.8 \%)$ \\
\hline $5-9$ years & $1099(24.8 \%)$ & $7478(20.7 \%)$ & $4792(14.9 \%)$ \\
\hline $10-14$ years & $757(17.1 \%)$ & $6231(17.2 \%)$ & $4856(15.1 \%)$ \\
\hline $15-19$ years & $794(17.9 \%)$ & $5700(15.7 \%)$ & $5003(15.5 \%)$ \\
\hline $20-24$ years & $870(19.7 \%)$ & $5135(14.2 \%)$ & $5450(16.9 \%)$ \\
\hline$\geq 25$ years & $531(12 \%)$ & $7181(19.8 \%)$ & $9602(29.8 \%)$ \\
\hline
\end{tabular}

$* p$-value $<0.05$ for robotic compared to open

${ }^{\wedge} p$-value $<0.05$ for robotic compared to laparoscopic 
Table 2 Surgeon characteristics

Surgeons performing Colectomy $(n=2487)$

Surgeons with IRU Surgeons with non-IRU

$(n=156,6.3 \%)$

$(n=2331,93.7 \%)$

Surgeon years of experience

10 or less

$53(34.0 \%)$

$566(24.3 \%)$

$11-20$

$58(37.2 \%)$

$654(28.1 \%)$

21 or more

$45(28.8 \%)$

$1111(47.7 \%)$

Colorectal surgery boarded

No

$99(63.5 \%)$

$2221(95.3 \%)$

Yes

$57(36.5 \%)$

$110(4.7 \%)$

Surgeons performing proctectomy $(n=1311)$

Surgeons with IRU Surgeons with non-IRU

$(n=94,7.2 \%)$ $(n=1217,92.8 \%)$

\section{Surgeon years of experience}

10 or less

$30(31.9 \%)$

$279(22.9 \%)$

$11-20$

$38(40.4 \%)$

$342(28.1 \%)$

21 or more

$26(27.6 \%)$

$596(48.9 \%)$

Colorectal surgery boarded

\begin{tabular}{lcc} 
No & $44(46.8 \%)$ & $1101(90.5 \%)$ \\
Yes & $50(53.2 \%)$ & $116(9.5 \%)$ \\
\hline
\end{tabular}

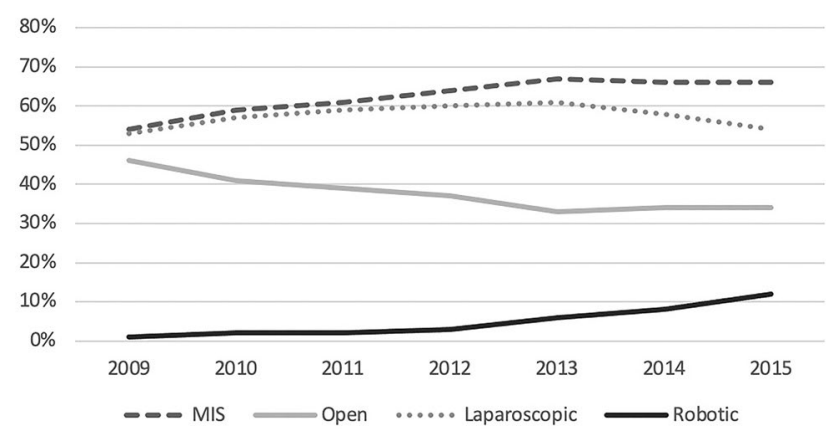

Fig. 2 Colectomy surgical approach over time

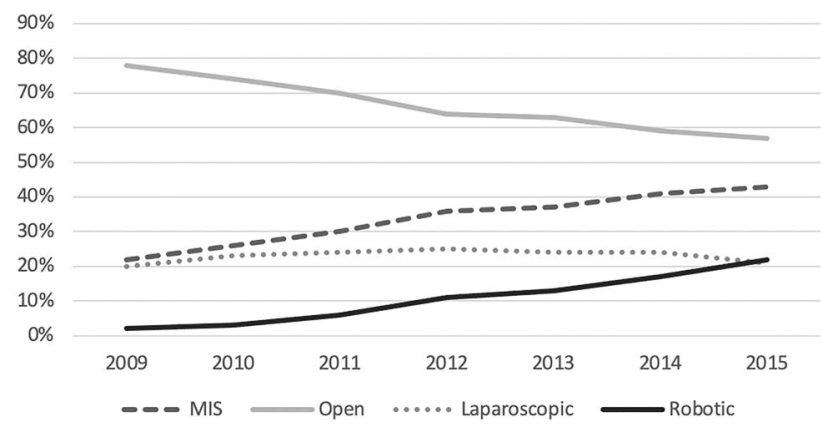

Fig. 3 Proctectomy surgical approach over time

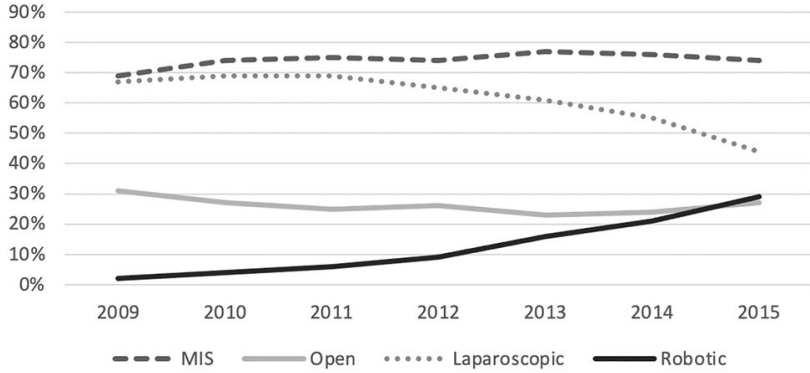

Fig. 4 Colectomy surgical approach over time among surgeons with increasing robotic utilization

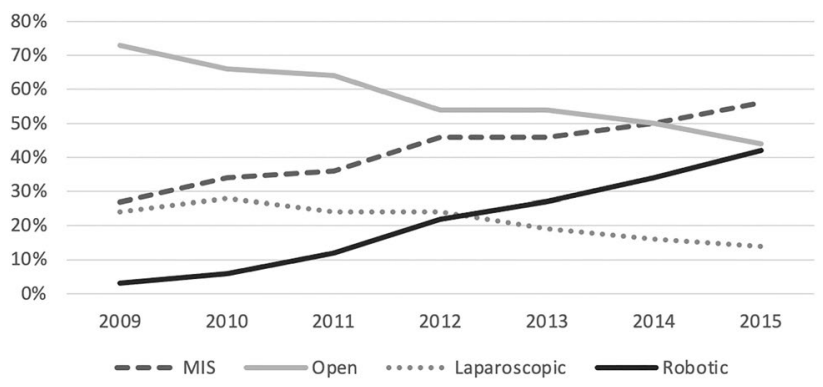

Fig. 5 Proctectomy surgical approach over time among surgeons with increasing robotic utilization 
Table 3 Multinomial regression: factors associated with surgical approach

\begin{tabular}{|c|c|c|c|}
\hline & Robotic & Lap & Open \\
\hline \multicolumn{4}{|l|}{ Surgery year } \\
\hline 2009-2012 & Ref & Ref & Ref \\
\hline $2013-2015$ & Ref & $0.242(0.214,0.265)$ & $0.180(0.163,0.202)$ \\
\hline \multicolumn{4}{|l|}{ Patient age } \\
\hline$\leq 60$ & Ref & Ref & Ref \\
\hline $61-74$ & Ref & $1.059(0.937,1.173)$ & $1.007(0.901,1.118)$ \\
\hline$\geq 75$ & Ref & $1.418(1.245,1.648)$ & $1.651(1.427,1.893)$ \\
\hline Female & Ref & Ref & Ref \\
\hline Male & Ref & $0.937(0.846,1.009)$ & $0.823(0.752,0.889)$ \\
\hline \multicolumn{4}{|c|}{ Medicaid insurance } \\
\hline No & Ref & Ref & Ref \\
\hline Yes & Ref & $1.005(0.825,1.237)$ & $1.528(1.220,1.918)$ \\
\hline \multicolumn{4}{|l|}{ Patient race } \\
\hline White & Ref & Ref & Ref \\
\hline Black & Ref & $1.084(0.916,1.271)$ & $0.992(0.882,1.172)$ \\
\hline Other & Ref & $1.099(0.922,1.277)$ & $0.980(0.875,1.132)$ \\
\hline \multicolumn{4}{|l|}{ Surgery } \\
\hline Proctectomy & Ref & Ref & Ref \\
\hline Colectomy & Ref & $7.070(6.507,8.077)$ & $1.155(1.037,1.273)$ \\
\hline \multicolumn{4}{|l|}{ ECS risk } \\
\hline Low & Ref & Ref & Ref \\
\hline Medium & Ref & $1.074(0.978,1.155)$ & $1.433(1.306,1.588)$ \\
\hline High & Ref & $1.073(0.998,1.153)$ & $2.209(2.004,2.382)$ \\
\hline \multicolumn{4}{|c|}{ Surgeon volume (tertile) } \\
\hline Low & Ref & Ref & Ref \\
\hline Med & Ref & $1.012(0.786,1.290)$ & $0.489(0.392,0.591)$ \\
\hline High & Ref & $1.354(1.024,1.612)$ & $0.288(0.224,0.341)$ \\
\hline \multicolumn{4}{|c|}{ Hospital volume (tertile) } \\
\hline Low & Ref & Ref & Ref \\
\hline Med & Ref & $0.553(0.453,0.651)$ & $0.596(0.483,0.715)$ \\
\hline High & Ref & $0.551(0.418,0.681)$ & $0.423(0.327,0.544)$ \\
\hline \multicolumn{4}{|l|}{ Rural hospital } \\
\hline No & Ref & Ref & Ref \\
\hline Yes & Ref & $0.709(0.369,1.970)$ & $1.485(0.67,3.754)$ \\
\hline \multicolumn{4}{|c|}{ Academic hospital } \\
\hline No & Ref & Ref & Ref \\
\hline Yes & Ref & $0.758(0.321,1.637)$ & $0.666(0.331,1.272)$ \\
\hline \multicolumn{4}{|c|}{ Colorectal surgery boarded } \\
\hline No & Ref & Ref & Ref \\
\hline Yes & Ref & $0.310(0.215,0.474)$ & $0.345(0.242,0.536)$ \\
\hline \multicolumn{4}{|c|}{ Surgeon years of experience } \\
\hline$<5$ years & Ref & Ref & Ref \\
\hline $5-9$ years & Ref & $0.624(0.504,0.744)$ & $0.906(0.749,1.203)$ \\
\hline $10-14$ years & Ref & $0.601(0.491,0.792)$ & $0.986(0.781,1.283)$ \\
\hline $15-19$ years & Ref & $0.338(0.247,0.498)$ & $0.702(0.533,1.046)$ \\
\hline $20-24$ years & Ref & $0.160(0.117,0.255)$ & $0.423(0.301,0.655)$ \\
\hline$\geq 25$ years & Ref & $0.190(0.144,0.291)$ & $0.689(0.517,0.985)$ \\
\hline
\end{tabular}


Fig. 6 Years of surgeon experience vs operative approach

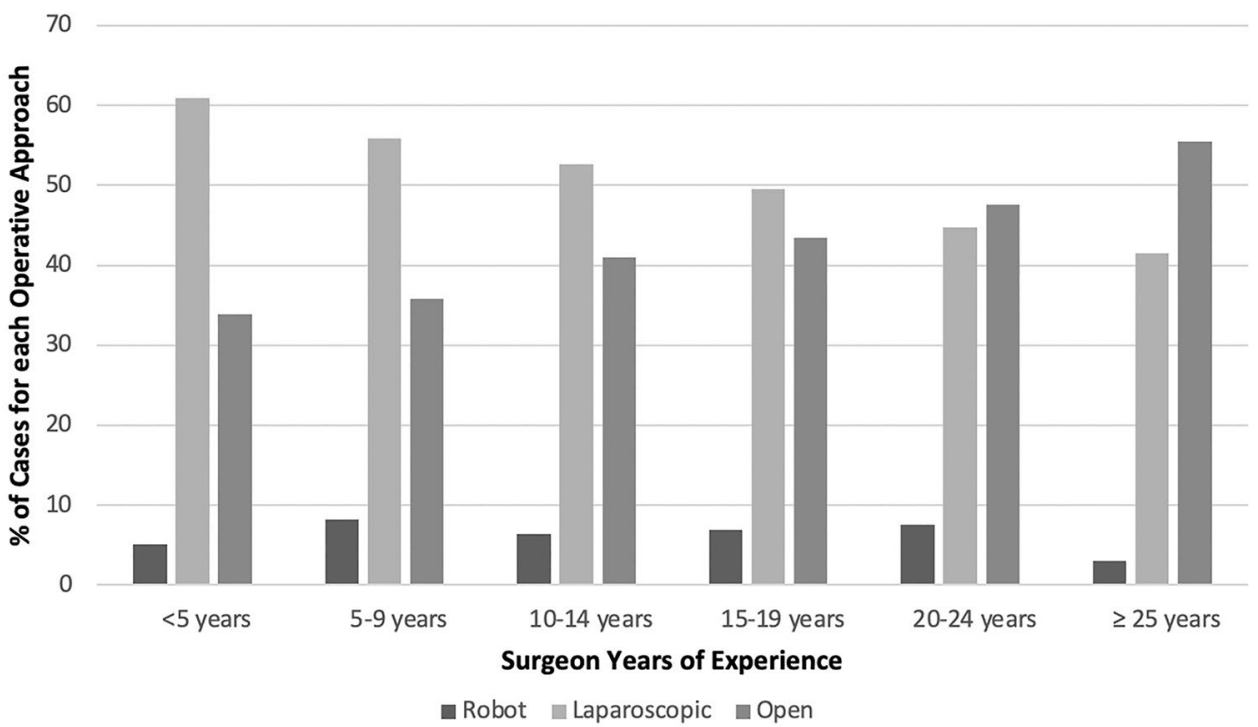

\section{Discussion}

In this population-based, all-payer study, the proportion of patients undergoing minimally invasive colorectal resections increased significantly from 2009 to 2015. Importantly, while increased robotic utilization was associated with some decreased laparoscopy use there was also a simultaneous decrease of open resections. This was observed for both colectomies and proctectomies; however, the increasing utilization of robotic surgery was far more pronounced for surgeons performing proctectomies. Together, these findings suggest that although the increased utilization of a robotic approach for colectomies is largely due to a transition from a laparoscopic approach to a robotic one, with similar colectomy MIS rates, the robotic approach appears to be superseding both laparoscopic and open approaches for proctectomies in real world practice, with increased proctectomy MIS rates.

These New York State, all-payer data support a recent nationwide Medicare study which concludes that robotic colectomy has largely replaced laparoscopic rather than open surgery when looking at hospital-level data [22]. However, given a stable rate of laparoscopic proctectomy over our study period ( $20 \%$ vs $21 \%$ ), robotic proctectomy has largely replaced open proctectomy with a decrease in open surgery from $78 \%$ of cases down to $43 \%$. The Robotic Versus Laparoscopic Resection for Rectal Cancer trial concluded that for cancer, robotic surgery does not confer an advantage over laparoscopic surgery [23]. However, the presented data would challenge that conclusion given that the robot allowed $35 \%$ more proctectomy cases to be approached with MIS. As suggested elsewhere, this change is likely due to the shorter learning curve and improved dexterity of the robot, compared to laparoscopy, particularly in pelvic surgery [24, 25].
This study has direct implications regarding quality of care delivery to patients and value of robotic surgery, particularly for rectal surgery, where robotic utilization is associated with the observed increase in MIS proctectomy rather than merely replacing laparoscopic cases which would have instead led to observing stable MIS [22, 26].

The robotic learning curve appears to also apply to older surgeons. Our group previously reported a strong inverse relationship between surgeon-years in practice and the rate of MIS colectomy utilization [27]. Our current data, however, would suggest that the relationship is not inverse if we look specifically at robotic surgery. The youngest surgeons perform robotic cases $5 \%$ of the time but this increases with surgeons who have practiced 5-9 years to $8 \%$. Furthermore, surgeons in practice for 20-24 years performed robotic cases $8 \%$ of the time as well. While this data are not conclusive, it does suggest that the robotic platform could possibly allow older surgeons to adopt and offer a minimally invasive approach more readily than laparoscopic surgery.

Our study has inherent limitations due to its retrospective nature and data source. There is certainly selection bias with regards to which patients received open versus laparoscopic versus robotic surgery, although the goal of the study was to describe the existing trends in surgical approach which are innately biased. Furthermore, our multinominal analysis has adjusted for the captured confounders. While SPARCS data are abstracted by trained medical records personnel, audited and verified, it remains at risk for miscoding. Nonetheless, there is no reason to believe that miscoding would be differential with regards to the study outcomes. Furthermore, SPARCS is limited to New York State and as such, its generalizability may not apply to all of the United States or abroad. Nevertheless, New York state is not too unlike other states with one 
largest metro area, a few smaller metro areas and extensive rural mileage. Moreover, our surgeon characteristics are limited to readily available facts such as years since completion of training and board certification. Data on previous laparoscopic or robotics specialty training or years of robotic usage are not readily available. Additionally, using SPARCS, we cannot discern whether surgeons with IRU versus non-IRU reflects a lack of surgeon access to robotic consoles or console time versus the choice to not utilize robotics. Furthermore, patient preference data are not collected in SPARCS, and as such, we cannot comment how patient preference influenced each individual operative approach nor how it influenced individual surgeons to utilize robotics or not. Additional factors which could drive operative approach choice are also not captured by our data; as such, our data are purely observational and we cannot demonstrate causation. Lastly, our data begins looking at surgeon proportional use in 2009; as such, if a surgeon maximized his/her robotic usage prior to our study period and had only steady usage thereafter, then he/she would not be labeled as a robotic user. However, given that at the onset of our study period only $1 \%$ of colectomies and $2 \%$ of proctectomies were performed robotically with limited patients in the robotic literature/series prior to 2009 , we expect that few surgeons, if any, are missed by our definition.

Notwithstanding these limitations, this is the first study to demonstrate an association between robotic surgery and increasing MIS proctectomy with a concurrent decrease in open proctectomy. While hospital-level data on robotic adoption's influence on MIS have previously been published and argue that there may be limited value with regards to robotic colectomy, surgeon-level data have not been published and proctectomy data have not been published. Here, we are able to demonstrate that during the study period, surgeons who had IRU increased their rate of MIS proctectomy by over $200 \%$ (27-56\% across study period) while those who had non-IRU only increased by about $50 \%$ (20-31\% over study period). Furthermore, this study is the first to demonstrate that at the surgeon-level a robotic approach does not markedly decline with years of experience, as does laparoscopy, but actually remains stable.

In conclusion, the rate of MIS for colorectal resections has significantly increased from 2009 to 2015 , particularly for proctectomies. The increase in MIS has occurred in the setting of overall stable laparoscopic cases and increased robotic cases, while surgeons with IRU specifically have had increased robotic cases with simultaneous decrease in both open and laparoscopic approaches. Older surgeons also have had IRU, and an inverse relationship between age and robotic usage does not seem to exist as it does for laparoscopy.
Acknowledgements This study was supported by the Research Foundation of the American Society of Colon and Rectal Surgeons' Research in Robotic Surgical Technology Grant.

Funding Research Foundation of the American Society of Colon and Rectal Surgeons' Research in Robotic Surgical Technology Grant.

\section{Declarations}

Disclosures Drs. Justiniano, Becerra, Loria, Xu, Aquina and Temple have no conflict of interest or financial ties to disclose. Dr. Fleming has author royalties from Up-to-Date which are not relevant to this submission.

\section{References}

1. McDermott KW, Freeman WJ, Elixhauser A (2006) Overview of operating room procedures during inpatient stays in U.S. Hospitals, 2014: Statistical Brief \#233. 2017 Dec. In: Healthcare Cost and Utilization Project (HCUP) Statistical Briefs [Internet]. Rockville (MD): Agency for Healthcare Research and Quality (US)

2. Jacobs M, Verdeja JC, Goldstein HS (1991) Minimally invasive colon resection (laparoscopic colectomy). Surg Laparosc Endosc 1(3):144-150

3. Fowler DL, White SA (1991) Laparoscopy-assisted sigmoid resection. Surg Laparosc Endosc 1(3):183-188

4. Franklin ME, Ramos R, Rosenthal D, Schuessler W (1993) Laparoscopic colonic procedures. World J Surg 17(1):51-56. https:// doi.org/10.1007/bf01655705

5. Schwenk W, Haase O, Neudecker J, Müller JM (2005) Short term benefits for laparoscopic colorectal resection. Cochrane Database Syst Rev. https://doi.org/10.1002/14651858.CD003145.pub2

6. Aziz O, Constantinides V, Tekkis PP et al (2006) Laparoscopic versus open surgery for rectal cancer: a meta-analysis. Ann Surg Oncol 13(3):413-424. https://doi.org/10.1245/ASO.2006.05.045

7. Lee Y, Fleming FJ, Deeb A-P, Gunzler D, Messing S, Monson JRT (2012) A laparoscopic approach reduces short-term complications and length of stay following ileocolic resection in crohn's disease: an analysis of outcomes from the NSQIP database. Colorectal Dis 14(5):572-577. https://doi.org/10.1111/j.1463-1318.2011.02756.x

8. Ohtani H, Tamamori Y, Arimoto Y, Nishiguchi Y, Maeda K, Hirakawa K (2012) A meta-analysis of the short- and long-term results of randomized controlled trials that compared laparoscopyassisted and open colectomy for colon cancer. J Cancer 3:49-57. https://doi.org/10.7150/jca.3621

9. Crawshaw BP, Chien H-L, Augestad KM, Delaney CP (2015) Effect of laparoscopic surgery on health care utilization and costs in patients who undergo colectomy. JAMA Surg 150(5):410-415. https://doi.org/10.1001/jamasurg.2014.3171

10. Aquina CT, Probst CP, Becerra AZ et al (2016) Missed opportunity: laparoscopic colorectal resection is associated with lower incidence of small bowel obstruction compared to an open approach. Ann Surg 264(1):127-134. https://doi.org/10.1097/ SLA.0000000000001389

11. Lee M-TG, Chiu C-C, Wang C-C et al (2017) Trends and outcomes of surgical treatment for colorectal cancer between 2004 and 2012- an analysis using national inpatient database. Sci Rep 7(1):1-8. https://doi.org/10.1038/s41598-017-02224-y

12. Osagiede O, Spaulding AC, Cochuyt JJ, Naessens J, Merchea A, Colibaseanu DT (2019) Trends in the use of laparoscopy and 
robotics for colorectal cancer in Florida. J Laparoendosc Adv Surg Tech A 29(7):926-933. https://doi.org/10.1089/lap.2019.0016

13. Gabriel E, Thirunavukarasu P, Al-Sukhni E, Attwood K, Nurkin SJ (2016) National disparities in minimally invasive surgery for rectal cancer. Surg Endosc 30(3):1060-1067. https://doi.org/10. 1007/s00464-015-4296-5

14. Fleshman J, Branda M, Sargent DJ et al (2015) Effect of laparoscopic-assisted resection vs open resection of stage II or III rectal cancer on pathologic outcomes: the ACOSOG Z6051 randomized clinical trial. JAMA 314(13):1346-1355. https://doi.org/10.1001/ jama.2015.10529

15. Tan BHL, Mytton J, Al-Khyatt W et al (2017) A comparison of mortality following emergency laparotomy between populations from New York State and England. Ann Surg 266(2):280-286. https://doi.org/10.1097/SLA.0000000000001964

16. Aquina CT, Blumberg N, Probst CP et al (2016) Large variation in blood transfusion use after colorectal resection: a call to action. Dis Colon Rectum 59(5):411-418. https://doi.org/10.1097/DCR. 0000000000000588

17. Aquina CT, Becerra AZ, Xu Z et al (2017) Nonelective colon cancer resection: a continued public health concern. Surgery 161(6):1609-1618. https://doi.org/10.1016/j.surg.2017.01.001

18. Quan H, Sundararajan V, Halfon P et al (2005) Coding algorithms for defining comorbidities in ICD-9-CM and ICD-10 administrative data. Med Care 43(11):1130-1139

19. van Walraven C, Austin PC, Jennings A, Quan H, Forster AJ (2009) A modification of the Elixhauser comorbidity measures into a point system for hospital death using administrative data. Med Care 47(6):626-633. https://doi.org/10.1097/MLR.0b013 e31819432e5

20. Thirumala PD, Muluk S, Udesh R et al (2017) Carotid artery disease and periprocedural stroke risk after transcatheter aortic valve implantation. Ann Card Anaesth 20(2):145-151. https://doi. org/10.4103/aca.ACA_13_17
21. Justiniano CF, Xu Z, Becerra AZ et al (2019) Effect of care continuity on mortality of patients readmitted after colorectal surgery. Br J Surg 106(5):636-644. https://doi.org/10.1002/bjs.11078

22. Sheetz KH, Norton EC, Dimick JB, Regenbogen SE (2020) Perioperative outcomes and trends in the use of robotic colectomy for medicare beneficiaries from 2010 through 2016. JAMA Surg 155(1):41-49. https://doi.org/10.1001/jamasurg.2019.4083

23. Jayne D, Pigazzi A, Marshall H et al (2017) Effect of roboticassisted vs conventional laparoscopic surgery on risk of conversion to open laparotomy among patients undergoing resection for rectal cancer: the ROLARR randomized clinical trial. JAMA 318(16):1569-1580. https://doi.org/10.1001/jama.2017.7219

24. Pigazzi A, Garcia-Aguilar J (2010) Robotic colorectal surgery: for whom and for what? Dis Colon Rectum 53(7):969-970. https:// doi.org/10.1007/DCR.0b013e3181db8055

25. Rashid TG, Kini M, Ind TEJ (2010) Comparing the learning curve for robotically assisted and straight stick laparoscopic procedures in surgical novices. Int J Med Robot 6(3):306-310. https://doi.org/ $10.1002 / \mathrm{rcs} .333$

26. Sheetz KH, Claflin J, Dimick JB (2020) Trends in the adoption of robotic surgery for common surgical procedures. JAMA Netw Open 3(1):e1918911-e1918911. https://doi.org/10.1001/jaman etworkopen.2019.18911

27. Aquina CT, Becerra AZ, Justiniano CF et al (2019) Surgeon, hospital, and geographic variation in minimally invasive colectomy. Ann Surg 269(6):1109-1116. https://doi.org/10.1097/SLA.00000 00000002694

Publisher's Note Springer Nature remains neutral with regard to jurisdictional claims in published maps and institutional affiliations. 